\title{
Nonlinear Dynamic Responses of a Honeycomb Sandwich Plate Subject to Transverse Excitations
}

\author{
Dongmei Wang ${ }^{1}$, Wei Zhang ${ }^{2}$, Minghui Yao ${ }^{2}$ and Yinli Liu ${ }^{1}$ \\ ${ }^{1}$ College of science, Tianjin University of Science and Technology, 300457 Tianjin, P. R. China \\ ${ }^{2}$ College of Mechanical Engineering, Beijing University of Technology, 100124 Beijing, P. R. China
}

\begin{abstract}
Nonlinear dynamic behaviors of a simply supported honeycomb sandwich plate subjected to the transverse excitations are investigated in this paper. Based on the classical thin plate theory and Von Karman large deformation theory, the governing equation of motion for the honeycomb sandwich plate is established by using the Hamilton principle. The nonlinear governing partial differential equation is discretized to the ordinary differential equations by differential quadrature method and then solved by Runge-Kutta-Fehlberg method. Based on the numerical simulations, combined with nonlinear dynamic theory, the influences of the frequency and amplitude of the transverse excitation are investigated respectively by using the bifurcation diagrams, Poincare maps and phase portraits. The results exhibit the existence of the period- 1 , period- 2 and chaotic responses with the variation of the excitations, which demonstrate that those motions appear alternately.
\end{abstract}

\section{Introduction}

As a kind of special composite structural material, sandwich beam and plates are widely used in a variety of aircraft structures and satellite launch vehicles. This is due to their many advantages such as low density, high strength ratio, high stiffness and ease of manufacture and repair compared with other structural materials. Therefore, sandwich structures have been received great attention, particularly in the recent decades.

A review of literature indicates that many analysis of sandwich plates, involving static, free vibration and stability analyzes, has been carried out. Using several plate models, Zenkour [1-2] performed a comprehensive analysis of functionally grade sandwich plates, including buckling, free vibration and stability analyzes. Li et al. [3] applied the three dimensional Ritz method based on the use of the Chebyshev polynomials for the free vibration analysis of functionally graded material sandwich plates. Dozio[4] studied the free vibration behavior of sandwich plates with FGM core via variable-kinematic 2-D Ritz models using Chebyshev polynomials. Neves et al.[5] combined quasi-3D higher-order shear deformation theories with meshless techniques in order to cope with bending, free vibration and stability analysis of FGM isotropic and sandwich plates. Fiorenzo [6,7] investigated free vibration and thermal stability of functionally graded sandwich plates and sandwich plates with anisotropic face sheets in thermal environment by using Hierarchical Trigonometric Ritz Formulation. Wang and Yuan [8] studied the effects of boundary conditions and different types loading on the displacement and stress distributions of the soft-core sandwich panels by using differential quadrature method.

The aforementioned research works provide the foundation and the guidance for us to study a variety of properties of the honeycomb sandwich plates. However, there is few research focused on the global bifurcations and chaotic dynamics of sandwich plates. We have found two papers on bifurcations and chaotic dynamics of sandwich plates. J.H.Zhang and W. zhang [9] investigated the global bifurcations and multi-pulse chaotic dynamics of a simply supported honeycomb sandwich rectangular plate under combined parametric and transverse excitations, they found that multi-pulse chaotic motions can occur in the honeycomb sandwich rectangular plate. Applying the Galerkin's approach, Zhang et al. [10] investigated nonlinear dynamic behaviors of a simply supported 3D-Kagome truss core sandwich plate subjected to the transverse and the inplane excitations. The bifurcation diagrams exhibit the existence of period, muti-period and chaotic motions appear alternately.

Usually, the honeycomb sandwich plates can be modeled by nonlinear partial differential equations. As we all know, in most cases it is difficult to obtain closedform solutions for nonlinear partial differential equations. As a result, people have to apply approximate, numerical or analytical methods to solve partial differential equations which arise in almost all engineering sphere. In the literature mentioned above, the approximate method used by scholars include finite difference method, finite element method, Ritz method, Galerkin method, 
homotopy analysis method, multiquadric collocations method and differential quadrature method.

The differential quadrature (DQ) method, which was introduced by Bellman [11] et al. in 1972, is a highly efficient approach to directly solving a partial differential equation for a finite domain with a set of given boundary conditions. The DQ method can discretize a finite domain by using a set of regularly or irregularly distributed grid points. Using appropriate interpolation polynomials, the unknown functions and their derivatives at an arbitrary point can be represented by a weighted linear combination of the functional values at all the discrete points. The accurate numerical results and little computational effort are obtained by using a smaller number of grid points.

The pioneering work on the application of the DQ method to the structural mechanics was given by Striz et al. $[12,13]$. Since the DQ method has lots of merits, such as simple principle, high precision, less computation and easy programming, this method has been used to solve a variety of problems in structural mechanics [14-20]. All these works demonstrated that the application of the DQ method caused the accurate results and less computational effort. However, most of the DQ applications are relevant to the problems of the static or free vibration in engineering fields, few applications are relevant to the problems of the nonlinear dynamics. The author [21] of this paper has applied the DQ method to the analysis of nonlinear dynamics of axially moving viscoelastic beams and the differential quadrature method was compared with other methods, which accuracy is verified.

In this paper, we developed the DQ method to analyze the nonlinear dynamical properties such as bifurcations and chaos of a simply supported honeycomb sandwich rectangular plate under transverse excitation for the first time. It is found from the numerical results that the DQ method can be successfully applied to reveal the complex dynamic phenomena of the honeycomb sandwich rectangular plate.

\section{Governing equation of the honeycomb sandwich plate}

Consider a simply supported honeycomb sandwich plate, where the edge lengths are $a$ and $b$ and $h$ the thickness is $h$, respectively. The honeycomb sandwich rectangular plate consists of 3 layers that made of two layers of thin skins and a hexagon honeycomb core. Based on the classical thin plate theory and Von Karman large deformation theory, by using the generalized Hamilton's principle, we have the governing equation of the nonlinear transverse vibration of the honeycomb sandwich plate under transverse load as following:

$$
\begin{gathered}
D_{11} w,_{x x x x}-\left(D_{12}-4 D_{66}-D_{21}\right) w,_{y y x x}-D_{22} w,_{y y y y} \\
+\left(A_{11}\left(w,_{x} w,_{x x}\right)+A_{12}\left(w,_{y} w,_{y x}\right)\right) w,_{x}+\left(A_{21}\left(w,_{x} w,_{y x}\right)+A_{22}\left(w,_{y} w,_{y y}\right)\right) w,_{y} \\
+\left(\mathrm{A}_{21}\left(\frac{1}{2} w,_{x}^{2}\right)+A_{22}\left(\frac{1}{2} w_{y}^{2}\right)\right) w,_{y y}+A_{66}\left(w,_{y y} w,_{x}+w,_{y} w,_{y x}\right) w,_{x}
\end{gathered}
$$

$$
\begin{aligned}
& +\left(A_{11}\left(\frac{1}{2} w_{x_{x}}^{2}\right)+A_{12}\left(w_{, y}^{2}\right)\right) w,_{x x}+A_{66}\left(w, x_{x x} w,_{y}+w, x_{x} w,_{y x}\right) w,_{y} \\
& -c \dot{w}+2 A_{66} w_{x} w_{y} w_{y x}+f=I_{0} \ddot{w}, \quad\{(x, y) \mid 0<x<a, 0<y<\} ،(1)
\end{aligned}
$$

Where a dot represents the partial differentiation with respect to time $t$, and a comma denotes the partial differentiation with respect to a specified coordinate, $c$ is a vibration damping coefficient, $f=F(x, y) \cos \Omega t$ is the transverse excitation that loads the plate

Where

$$
\left(A_{i j}, D_{i j}\right)=\sum_{k=1}^{3} \int_{z_{k}}^{z_{k+1}} Q_{i j}^{k}\left(1, z^{2}\right) d z, \quad(i, j=1,2,6)
$$

$Q_{i j}^{k}$ is the conversion elastic constant of layer $k$

$$
Q_{11}^{k}=\frac{E_{1}^{k}}{1-v_{12}^{k} v_{21}^{k}}, Q_{12}^{k}=\frac{v_{12} E_{1}^{k}}{1-v_{12}^{k} v_{21}^{k}}, Q_{22}^{k}=\frac{E_{2}^{k}}{1-v_{12}^{k} v_{21}^{k}}, Q_{66}^{k}=G_{12}^{k}
$$

where $E_{1}=E_{2}, v_{12}=v_{21}$.

$I_{0}$ is the quality of honeycomb sandwich plates and

$$
I_{0}=\sum_{k=1}^{3} \int_{z_{k}}^{z_{k+1}} \rho_{k} d z
$$

The boundary conditions are

$$
\begin{aligned}
& x=0 \text { or } x=a, w=0, \frac{\partial^{2} w}{\partial x^{2}}=0, \\
& y=0 \text { or } y=b, w=0, \frac{\partial^{2} w}{\partial y^{2}}=0 .
\end{aligned}
$$

\section{Differential quadrature analogs}

The DQM formulation can be found in literature [22]. For integrity and convenience, this subsection reviews the main idea of the DQM. The idea of the DQM is to directly compute the derivative of a function at any grid point within its bounded domain by estimating a weighted linear sum of values of the function at a small set of points belonging to the domain.

Introduce $M \times N$ unequally spaced sampling points as

$$
\begin{aligned}
& x_{i}=\left(\frac{a}{2}\right)\left[1-\cos \frac{(i-1) \pi}{N-1}\right], \quad(i=1,2, \cdots, M), \\
& y_{j}=\left(\frac{b}{2}\right)\left[1-\cos \frac{(j-1) \pi}{N-1}\right], \quad(j=1,2, \cdots, N) .
\end{aligned}
$$

Applying the quadrature rules for the derivatives of a function at the sampling points yield

$$
\begin{gathered}
w_{, x}\left(x_{i}, y_{j}, t\right)=\sum_{k=1}^{M} a_{i k}^{(1)} w\left(x_{k}, y_{j}, t\right), \\
w_{, x x}\left(x_{i}, y_{j}, t\right)=\sum_{k=1}^{M} a_{i k}^{(2)} w\left(x_{k}, y_{j}, t\right), \\
w_{, x x c x}\left(x_{i}, y_{j}, t\right)=\sum_{k=1}^{M} a_{i k}^{(4)} w\left(x_{k}, y_{j}, t\right), \\
w_{, y}\left(x_{i}, y_{j}, t\right)=\sum_{s=1}^{N} a_{j s}^{(1)} w\left(x_{i}, y_{s}, t\right), \\
w_{, y y}\left(x_{i}, y_{j}, t\right)=\sum_{s=1}^{N} a_{j s}^{(2)} w\left(x_{i}, y_{s}, t\right),
\end{gathered}
$$




$$
\begin{gathered}
w_{, y y y y}\left(x_{i}, y_{j}, t\right)=\sum_{s=1}^{N} a_{j s}^{(4)} w\left(x_{i}, y_{s}, t\right), \\
w_{, x y}\left(x_{i}, y_{j}, t\right)=\sum_{s=1}^{N} a_{j s}^{(1)} \sum_{k=1}^{M} a_{i k}^{(1)} w\left(x_{k}, y_{s}, t\right), \\
w_{, x x y y}\left(x_{i}, y_{j}, t\right)=\sum_{s=1}^{N} a_{j s}^{(2)} \sum_{k=1}^{M} a_{i k}^{(2)} w\left(x_{k}, y_{s}, t\right) . \\
i, k=1,2, \cdots, M ; j, s=1,2, \cdots, N \\
w_{, y y}\left(x_{i}, y_{j}, t\right), w_{, y y y}\left(x_{i}, y_{j}, t\right), w_{, x y}\left(x_{i}, y_{j}, t\right), w_{, x x y y}\left(x_{i}, y_{j}, t\right) \text { are the }
\end{gathered}
$$
first, the second and the forth-order partial derivative approximations of $x, y$ at the nth time level at the grid point $\left(x_{i}, y_{j}\right)$, respectively, and the DQM weighting coefficients are the expression

$$
\begin{aligned}
& a_{i j}^{(1)}=\frac{\prod_{k=1, k \neq i}^{N}\left(x_{i}-x_{k}\right)}{\left(x_{i}-x_{j}\right) \prod_{k=1, k \neq j}^{N}\left(x_{i}-x_{j}\right)},(i, j=1,2, \cdots, N ; j \neq i), \\
& a_{i j}^{(m)}=m\left[a_{i i}{ }^{(m-1)} a_{i j}^{(1)}-\frac{a_{i j}^{(m-1)}}{x_{i}-x_{j}}\right], \quad a_{i i}{ }^{(m)}=-\sum_{k=1, k \neq i}^{N} a_{i k}{ }^{(m)} . \\
& (m=2,3, \cdots ; \quad i, j=1,2, \cdots N ; j \neq i)
\end{aligned}
$$

In this paper, set $a=b=1, M=N$, and the coefficient matrix ${ }^{\left(a_{i j}{ }^{(m)}\right)}$ of the first order, second order and fourth order derivative are expressed in $A B 1, A B 2, A B 4$, respectively and set the internal node function value vector $\left[w\left(x_{1}, y_{1}, t\right), w\left(x_{2}, y_{2}, t\right), \cdots, w\left(x_{N-2}, y_{N-2}, t\right)\right]$ is expressed in $W$. Modification of the weighting coefficient matrix [22] to implement the boundary condition equations (5), yields

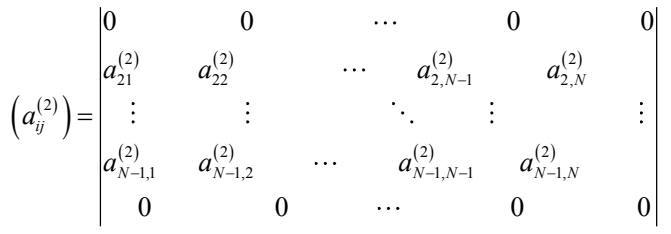

With ${ }^{\left(a_{i j}^{(2)}\right)}$, the modified weighting coefficient matrix $\left(a_{i j}^{(4)}\right)$ can be computed by using the matrix multiplication method. Substitution of equations (7) into equations (1) yields

$$
\begin{aligned}
& D_{11} \times(I \otimes A B 1 \times W)-\left(D_{11}-4 D_{66}-D_{21}\right) \times(A B 2 \otimes I \times(I \otimes A B 2 \times W)) \\
& -D_{22} \times(A B 4 \otimes I \times W)+\left[A_{21}(I \otimes A B 1 \times W) \bullet(A B 1 \otimes I \times(I \otimes A B 1 \times W))\right. \\
& \left.+A_{22}(A B 1 \otimes I \times W) \bullet(A B 2 \otimes I \times W)\right] \bullet(A B 1 \otimes I \times W)+\left[A_{21}\left(\frac{1}{2}\right)\right. \\
& \left.(I \otimes A B 1 \times W) \bullet(I \otimes A B 1 \times W)+A_{22}\left(\frac{1}{2}\right)(A B 2 \otimes I \times W) \bullet(A B 2 \otimes I \times W)\right] \\
& \bullet(A B 2 \otimes I \times W)+\left[A_{11}(I \otimes A B 1 \times W) \bullet(I \otimes A B 2 \times W)+A_{12}(A B 1 \otimes I \times W)\right. \\
& \bullet(A B 1 \otimes I \times(I \otimes A B 1 \times W))] \bullet(I \otimes A B 1 \times W)+A_{66}[(A B 2 \otimes I \times W) \\
& \bullet(I \otimes A B 1 \times W)+(A B 1 \otimes I \times W) \bullet(A B 1 \otimes I \times(I \otimes A B 1 \times W))] \bullet(A B 1 \otimes I \times W)
\end{aligned}
$$

$$
\begin{aligned}
& {\left[A_{11}\left(\frac{1}{2}\right)(I \otimes A B 1 \times W) \bullet(I \otimes A B 1 \times W)+A_{12}(A B 2 \otimes I \times W) \bullet(A B 2 \otimes I \times W)\right]} \\
& \bullet(A B 1 \otimes I \times W)+\left[A_{11}\left(\frac{1}{2}\right)(I \otimes A B 1 \times W) \bullet(I \otimes A B 1 \times W)\right. \\
& \left.+A_{12}(A B 2 \otimes I \times W) \bullet(A B 2 \otimes I \times W)\right] \bullet(I \otimes A B 2 \times W) \\
& +A_{66}[(A B 1 \otimes I \times W) \bullet(I \otimes A B 2 \times W)+(I \otimes A B 1 \times W) \\
& \bullet(A B 1 \otimes I \times(I \otimes A B 1 \times W))] \bullet(A B 1 \otimes I \times W)-c \dot{W}+2 A_{6 \circ}[(I \otimes A B 1 \times W) \\
& \bullet(A B 1 \otimes I \times W)] \bullet(A B 1 \otimes I \times(I \otimes A B 1 \times W))+f=I_{0} \ddot{W}
\end{aligned}
$$

Where $I$ represent a $(N-2) \times(N-2)$ unit matrix, “. "means that two vectors are multiplied by the corresponding elements, “ $x, \otimes$ ” represent vector product and Kronecker product respectively. Equations (11) includes $(N-2) \times(N-2)$ nonlinear ordinary differential equations with unknown transverse displacements vector $W$. Given initial conditions, for a set of given parameters $E, \Omega, F$ and so on. Then equation (11) can be numerically solved by Runge-Kutta-Fehlberg method, and the transverse displacement $w\left(x_{i}, y_{i}, t\right)$ at each $t$ can be obtained.

\section{Numerical results}

In the following, the DQM is applied to the governing equations (1) to investigate the effect of the frequency and the amplitude of the transverse excitation on the nonlinear dynamic behaviors of the honeycomb sandwich plate. The bifurcation diagram is a modern technique used to analyze nonlinear systems. To give the bifurcation diagrams, we will project the Poincare maps onto the displacement axis and the velocity axis. By varying the transverse excitation frequency $\Omega$ and the amplitude $F$, the two types of bifurcation diagrams are respectively presented with all other parameters fixed as shown Figure 1 and Figure 2. In order to illustrate in detail the bifurcation behaviors, the Poincare maps which consists of the displacements $w$ and the velocity $\frac{d w}{d t}$, and the phase portraits which consists of the displacement $w$ and the velocity $\frac{d w}{d t}$ are respectively depicted.

In all present investigations, the center of the plate is chosen to represent the motion of the plate, the initial conditions are

$$
w(x, y, 0)=0, w_{t}(x, y, 0)=0.01
$$

Figure1 presents the bifurcation diagrams for the displacements $w$ via the transverse excitation frequency $\Omega$ when the transverse excitation amplitude $F=3000 \mathrm{~N}$

From Figure 1, we can see that when the frequency $\Omega$ is located in the interval $[62,63] \mathrm{Hz}$, the system movement is complicated chaotic motions. With the increasing of the frequency up to $67 \mathrm{~Hz}$, most of the motions are simple periodic motions which interrupted by two small chaotic 
windows, when $\Omega=64.475 \mathrm{~Hz}$, period doubling bifurcation appears, that is the single period motions change into period-2 motions. With the further increase of the frequency of the transverse excitation, the system movement change into chaotic motions again until the last.

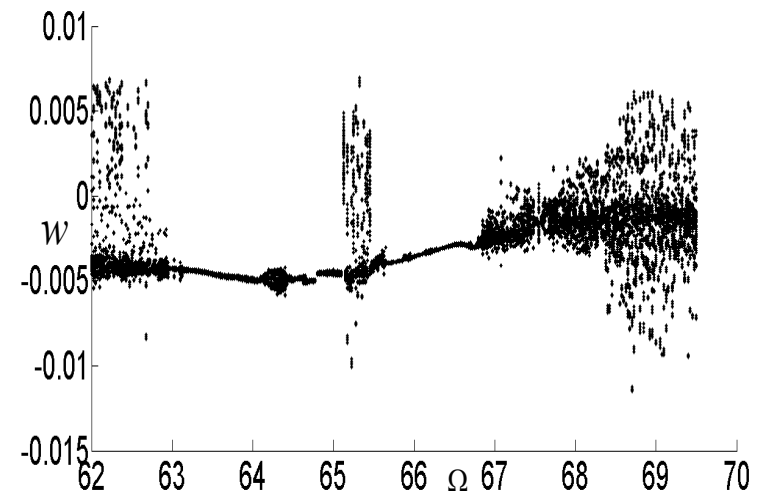

Figure 1. Effect of transverse excitation frequency: bifurcation diagrams for the displacement $w$ via $\Omega$.

Figure2 presents the bifurcation diagrams for the displacements $w$ via the transverse excitation amplitude fluctuation $F$ in $[2500,2900] \mathrm{N}$, when $\Omega=62 \mathrm{~Hz}$. From Figure 2, we can see that chaotic and periodic motions appear alternately when the transverse excitation amplitude $F$ is located in $[2500,2662] \mathrm{N}$, which most of the motions are large amplitude chaotic motions. Increasing the amplitude of the transverse excitation $F$, it is observed that a large window periodic motions appear and there is a period doubling bifurcation when $F=2846 \mathrm{~N}$. With the further increase of the transverse excitation amplitude from $F=2850 \mathrm{~N}$, the system movement change into chaotic motions again until the last.

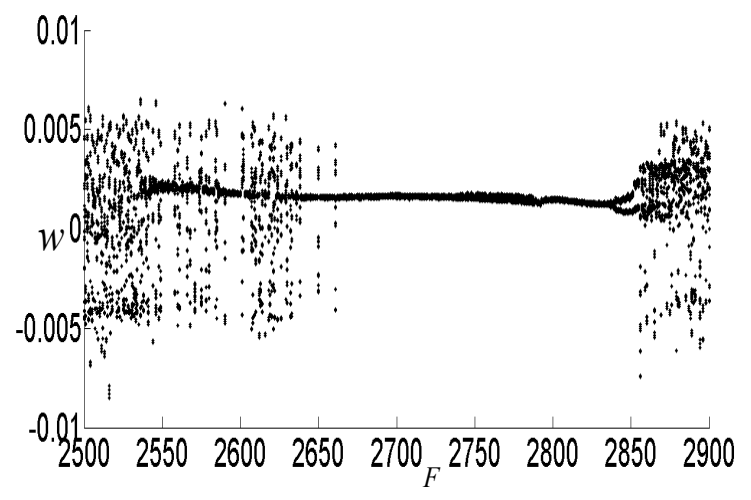

Figure 2.Effect of amplitude of the transverse excitation: bifurcation diagrams for the displacement ${ }^{w}$ via $F$

Typical periodic motion and chaotic motion are presented in figures 3-5, in terms of (a) phase portrait of ${ }^{w}$ and $\dot{w},(\mathrm{~b})$ the Poincare maps of ${ }^{w}$ and ${ }^{\dot{w}}$. They can be easily differentiated. In a projection of a Poincare map, periodic-1 motion is represented by one point, periodic- 2 motion is represented by two point and chaos is represented by infinite number of points.

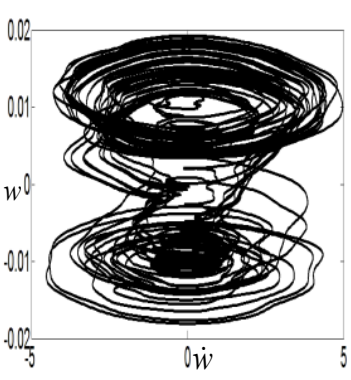

(a)

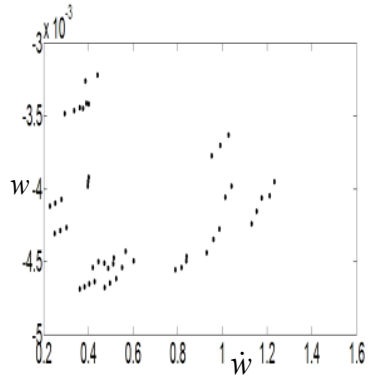

(b)
Figure 3. Chaotic motion appears when $F=3000 \mathrm{~N}$, $\Omega=62.5 \mathrm{~Hz}$

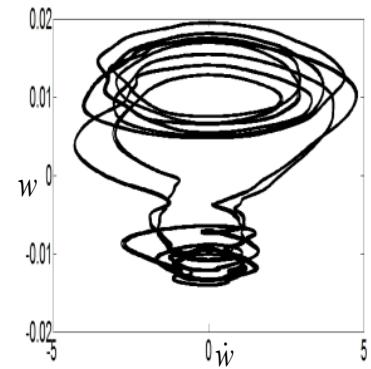

(a)

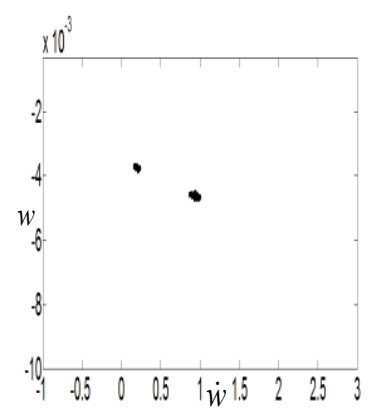

(b)
Figure 4. Period-2 motion appears when $F=2846 \mathrm{~N}$, $\Omega=62 \mathrm{~Hz}$

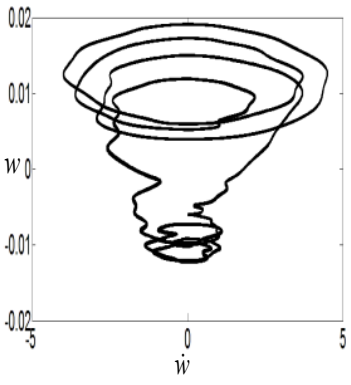

(a)

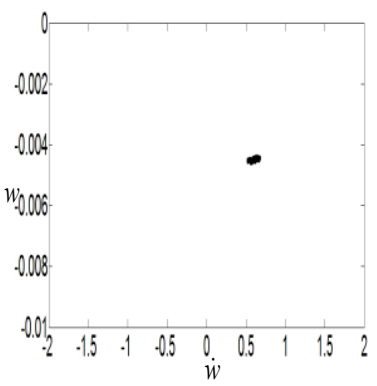

(b)
Figure 5. Period-1 motion appears when $F=2700 \mathrm{~N}$, $\Omega=62 \mathrm{~Hz}$.

\section{Summary}

This paper studies the bifurcations and chaotic motions for a simply supported honeycomb sandwich rectangular plate under transverse excitation which governing equation is established by using the generalized Hamilton's principle. The DQ method is developed to solve the nonlinear partial-differential governing equation numerically. Based on the numerical solutions, the nonlinear dynamical behaviors are numerically 
investigated by means of the Poincare map, the phase portraits. Two types of bifurcation diagrams are presented to show the displacement and velocity projections of the Poincare maps changing with the frequency and the amplitude of the transverse excitation. The results show that the chaotic motion and the periodic motion exchange alternately for a sufficiently large fluctuation of frequency and the amplitude of the transverse excitation. In addition, comparing the phase portraits with the Poincare map, the nonlinear dynamical behaviors are identified by the phase portraits are the same as the results obtained using the Poincare map. These results show the DQ method is an efficient method for the nonlinear analysis of honeycomb sandwich plate.

\section{References}

1. A. M. Zenkour, A comprehensive analysis of functionally graded sandwich plates:part 2-Buckling and free vibration, Int J Solids Struct. 42, 5243-5258 (2005).

2. A. M. Zenkour, M. Sobhy, Thermal buckling of various types of FGM sandwich plates, Compos Struct. 93(1), 93-112 (2010)

3. Q. Li, V.P. Iu, K.P. Kou, hree-dimensional vibration analysis of functionally graded material sandwich plates, J Sound Vib. 311, 498-515 (2008)

4. L.Dozio, Natural frequencies of sandwich plates with FGM core via variable-kinematic 2-D Ritz models, Compos Struct. 96 , 561-568 (2013)

5. A.M.A.Neves, A.J.M.Ferreira, E.Carrera, M. Cinefra, C.M.C. Roque, R.M.N.Jorge, et al., Static, free vibration and buckling analysis of analysis of isotropic and sandwich functionally graded plates using a quasi-3D higher-order shear deformation theory and a meshless technique, Compos Part B: Eng. 44(1), 657-674 (2013)

6. Fiorenzo A. Fazzolari, Erasmo Carrera, Free vibration analysis of sandwich plates with anisotropic face sheets in thermal environment by using the hierarchical trigonometric Ritz formulation, Composites: Part B 50, 67-81 (2013)

7. Fiorenzo A. Fazzolari, Natural frequencies and critical temperatures of functionally graded sandwich plates subjected to uniform and non-uniform temperature distributions, Composite Structures 121, 197-210 (2015)

8. X.W.Wang, Z.X.Yuan, Accurate stress analysis of sandwich panels by differential quadrature method, Applied Mathematical Modeling 43, 548-565 (2017)

9. J. H .Zhang, and W. Zhang, Multi-pulse chaotic dynamics of non-autonomous nonlinear system for a honeycomb sandwich plate, Acta Mechanica 223, 1047-1066 (2012)
10. W. Zhang, J. E.Chen., D. X.Cao, L.H. Chen, Nonlinear dynamic responses of a truss core sandwich plate. Composite Structures 108, 367-386 (2014)

11. R.Bellman, B.G.Kashef, J.Casti, Differential quadrature: a technique for the rapid solution of nonlinear partial differential equations. Journal of Computational Physics 10, 40-52 (1972)

12. C.W. Bert, S.K. Jang, A.G.Striz, Two new approximate methods for analyzing free vibration of structural components, AIAA Journal 26, 612-618 (1988)

13. C.W. Bert, S.K. Jang, A.G.Striz, Nonlinear bending analysis of orthotropic rectangular plates by the method of differential quadrature, Computational Mechanics 5, 217-226 (1989)

14. P.Malekzadeh, G.Karami, Differential quadrature nonlinear analysis of skew composite plates based on FSDT, Engineering Structures 28, 1307-1318 (2006)

15. A.Alibeigloo, R.Madoliat, Static analysis of crossply laminated plates with integrated surface piezoelectric layers using differential quadrature, Composite Structures 88, 342-353 (2009)

16. J.E.Jam, S. Maleki, A.Andakhshideh, Non-linear bending analysis of moderately thick fundtionally graded plates using generalized differential quadrature method, International Journal of Aerospace Sciences 1, 49-56 (2011)

17. P.Li, Y.R.Yang, L.Lu, Instability analysis of twodimensional thin panels in subsonic flow with differential quadrature method, Journal of Dynamics and Control 10, 11-14 (2012)

18. Y.F.Zhou, Z.M.Wang, Application of the differential quadrature method to free vibration of viscoelastic thin plate with linear thickness variation, Meccanica 49, 2817-2828 (2014)

19. B.Laxmi, S.Chakraverty, Application of Differential Quadrature method in free vibration analysis of nanobeams based on various nonlocal theories, Computers and Mathematics with Applications 69, 1444-1462 (2015)

20. X.W.Wang, Z.X.Yuan, Accurate stress analysis of sandwich panels by the differential quadrature method, Applied Mathematical Modelling 43, 548565, (2017)

21. W. Zhang, D.M.Wang, M.H.Yao, Using fourier differential quadrature method to analyze transverse nonlinear vibrations of an axially accelerating viscoelastic beam, Nonlinear Dyn 78, 839-856 (2014)

22. C.Shu, Differential Quadrature and its Application in Engineering. (Springer, Berlin 2000) 